\title{
A THEOREM OF BEURLING AND TSUJI IS BEST POSSIBLE
}

\author{
SHINJ YAMASHITA
}

\begin{abstract}
We shall show that Beurling-Tsuji's theorem (see Theorem A) is, in a sense, best possible. For each pair $a, b \in(0,+\infty)$ there exists a function $f$ holomorphic in $\{|z|<1\}$ such that the Euclidean area of the Riemannian image of each non-Euclidean disk of non-Euclidean radius $a$, is bounded by $b$, and such that $f$ has finite angular limit nowhere on the unit circle.
\end{abstract}

1. Introduction. Let $D=\{|z|<1\}$, and let $\Gamma=\{|z|=1\}$. For a function $f$ holomorphic in $D$, and for a subset $E$ of $D$ we use the notation

$$
A(E, f)=\iint_{E}\left|f^{\prime}(z)\right|^{2} d x d y, \quad z=x+i y .
$$

The following theorem is due to A. Beurling and M. Tsuji.

TheOREM A ([1], [4], see [5, Theorem VIII.49, p. 344]). Let $f$ be a function holomorphic in $D$ with $A(D, f)<+\infty$. Then $f$ has a finite angular limit at each point of $\Gamma$ except for a set of zero logarithmic capacity.

We shall show that extensions of Theorem A are, in a sense, impossible.

Consider the non-Euclidean hyperbolic metric

$$
\sigma(w, z)=\frac{1}{2} \log \frac{|1-\bar{z} w|+|z-w|}{|1-\bar{z} w|-|z-w|}, \quad z, w \in D,
$$

to define

$$
H(z, a)=\{w \in D ; \sigma(w, z)<a\}, \quad z \in D, a \in(0,+\infty] .
$$

We let $F(a, b)$ be the family of all holomorphic functions $f$ in $D$ such that, for each $z \in D, A(H(z, a), f) \leqslant b$, where $a \in(0,+\infty]$ and $b \in(0,+\infty)$. Then, $f$ of Theorem A belongs to $F(+\infty, b)$ with $b=A(D, f)$.

TheOREM 1. Given $a \in(0,+\infty)$ and $b \in(0, \infty)$, we may find $f \in F(a, b)$ such that neither $\operatorname{Re} f$ nor $\operatorname{Im} f$ has a finite angular limit at any point of $\Gamma$.

Thus, $f$ has not a finite angular limit at any point of $\Gamma$.

2. Bloch function. A function $f$ in $D$ is called Bloch [3] if $f$ is holomorphic in $D$ and if

$$
\beta(f)=\sup _{z \in D}\left(1-|z|^{2}\right)\left|f^{\prime}(z)\right|<+\infty .
$$

Let $B(c)$ be the family of all Bloch functions $f$ with $\beta(f) \leqslant c, c \in(0,+\infty)$.

Received by the editors December 21, 1977 and, in revised form, February 27, 1978.

AMS (MOS) subject classifications (1970). Primary 30A72. 
TheOREM 2. (2.1) If $a \in(0,+\infty)$, then each $f \in B(c)$ is a member of $F(a, b)$ with

$$
b=\pi c^{2} p(a)^{2} /\left(1-p(a)^{2}\right)
$$

where

$$
p(a)=\left(e^{2 a}-1\right) /\left(e^{2 a}+1\right) .
$$

(2.2) If $a \in(0,+\infty], b \in(0,+\infty)$, and if $f \in F(a, b)$, then $f \in B(c)$ with

$$
c^{2}=b /\left[\pi p(a)^{2}\right] \quad(p(+\infty)=1) .
$$

Proof. (2.1) Since

$$
\left|f^{\prime}(w)\right| \leqslant c\left(1-|w|^{2}\right)^{-1}, \quad w \in D,
$$

it follows that, for each $z \in D$,

$$
\begin{aligned}
A(H(z, a), f) \leqslant & c^{2} \iint_{H(z, a)}\left(1-|w|^{2}\right)^{-2} d x d y \\
& =c^{2} \iint_{|w|<p(a)}\left(1-|w|^{2}\right)^{-2} d x d y=b \quad(w=x+i y),
\end{aligned}
$$

because $\left(1-|w|^{2}\right)^{-2} d x d y$ is invariant under non-Euclidean transformations.

(2.2) Set $p=p(a)$, and for each fixed $z \in D$, set

$$
g(w)=f((p w+z) /(1+\bar{z} p w)), \quad w \in D .
$$

Then

$$
\pi p^{2}\left(1-|z|^{2}\right)^{2}\left|f^{\prime}(z)\right|^{2}=\pi\left|g^{\prime}(0)\right|^{2} \leqslant A(D, g)=A(H(z, a), f) \leqslant b .
$$

Therefore, $\beta(f) \leqslant c$, whence $f \in B(c)$.

3. Proof of Theorem 1. We shall make use of the two lemmata due to P. A. Lappan:

LEMMA 1 [2, p. 113]. There exists a holomorphic function $g$ in D, satisfying

$$
\sup _{z \in D}(1-|z|)|g(z)| \leqslant 2,
$$

and

$$
\limsup _{0<r \rightarrow 1-}(1-r)|g(r \zeta)|>0
$$

for each $\zeta \in \Gamma$.

LeMma 2 [2, Lemma 3]. Let $f$ be a holomorphic function in $D$ such that

$$
\limsup _{0<r \rightarrow 1-}(1-r)\left|f^{\prime}(r \zeta)\right|>0
$$

at a point $\zeta \in \Gamma$. Then, neither $\operatorname{Re} f$ nor $\operatorname{Im} f$ has a finite angular limit at $\zeta$.

We note that our Lemma 2 is worded differently than Lemma 3 of [2], but the content is equivalent.

For the proof of Theorem 1 we choose $k>0$ such that 


$$
k^{2}=16 \pi p(a)^{2} /\left[b\left(1-p(a)^{2}\right)\right] .
$$

Let $f$ be a function holomorphic in $D$ such that $f^{\prime}=k^{-1} g$, where $g$ is the function in Lemma 1. Then it follows from (3.1) that $f \in B(c)$ with $c=4 k^{-1}$. It follows from Theorem 2, (2.1), together with (3.4) that $f \in F(a, b)$. It further follows from (3.2) that (3.3) is true at every point $\zeta$ of $\Gamma$. Thus, our assertion on the angular limits of $\operatorname{Re} f$ and $\operatorname{Im} f$ follows from Lemma 2.

\section{REFERENCES}

1. A. Beurling, Ensembles exceptionnelles, Acta Math. 72 (1940), 1-13.

2. P. A. Lappan, Fatou points of harmonic normal functions and uniformly normal functions, Math. Z. 102 (1967), 110-114.

3. C. Pommerenke, On Bloch functions, J. London Math. Soc. (2) 2 (1970), 689-695.

4. M. Tsuji, Beurling's theorem on exceptional sets, Tôhoku Math. J. 2 (1950), 113-125.

5. , Potential theory in modern function theory, Maruzen, Tokyo, 1959.

Department of Mathematics, Tokyo Metropolitan University, Fukazawa, Setagaya-kU, Tokyo 158, JAPAN 